\title{
Influence of bridged monomer on porosity and sorption properties of mesoporous silicas functionalized with diethylenetriamine groups
}

\author{
Mariusz Barczak $^{1}$ D $\cdot$ Małgorzata Gil $^{1} \cdot$ Konrad Terpiłowski $^{1} \cdot$ Daniel Kamiński $^{1} \cdot$ Piotr Borowski $^{1}$
}

Received: 12 January 2019 / Revised: 22 February 2019 / Accepted: 25 February 2019 / Published online: 7 March 2019

(c) The Author(s) 2019

\begin{abstract}
Mesoporous organosilicas functionalized simultaneously with both pendant amine groups and ethylene/phenylene bridges, were synthesized to evaluate the effect of the bridges incorporated in the silica framework on the structure, porosity and adsorption properties of final materials. It turned out that the presence of the bridges enhanced the formation of porosity but adversely affected the efficiency of amination. DFT theoretical calculation showed that the amine groups can be partially bonded by phenyl groups of the bridges. The obtained materials were tested as sorbents of diclofenac, which is considered

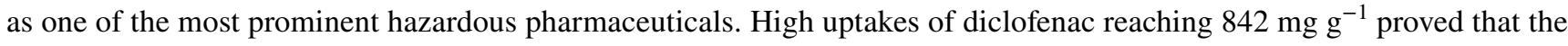
obtained materials could be applied for removal of drugs from waters and wastewaters, particularly when high concentrations of pharmaceutical are considered. For the phenylene bridged-based materials the diclofenac adsorption occurs only in the pores while for the others also on the external surface as indicated by different diclofenac desorption kinetics-this observation can be used to modulate the releasing profiles of drugs via proper design of the surface.
\end{abstract}

Keywords Mesoporous silica $\cdot$ Hybrid materials $\cdot$ Porosity $\cdot$ Adsorption $\cdot$ Diclofenac $\cdot$ Controlled release

\section{Introduction}

Templated mesoporous silicas are frequently studied adsorbents due to the wide range of beneficial properties including high surface areas, tunable pore sizes and good hydrothermal and mechanical stability. Two most known representatives are MCM-41 (Beck et al. 1992; Kresge et al. 1992) and SBA-15 (Schmidt-Winkel et al. 1999; Zhao et al. 1998) structures, both possessing hexagonal arrangement of cylindrical mesopores. They are synthesized using different templates and under different conditions. Apart from structural and morphological features that can be controlled by proper design of the fabrication protocol also chemical properties can be simultaneously tailored. Remarkable development of different modification protocols has been reported in the literature which brought the mesoporous silicas closer to the applications in which precise and simultaneous control over the surface chemistry, porous structure and stability is required. Those applications include for example controlled

Mariusz Barczak

mbarczak@umcs.pl

1 Faculty of Chemistry, Maria Curie-Sklodowska University, Maria Curie-Sklodowska Sq. 3, 20-031 Lublin, Poland drug delivery (Martín et al. 2018; Song et al. 2005; Vallet-Regí et al. 2017), wastewater treatment (Aguado et al. 2009; Barczak 2018; Cotea et al. 2012; Huang et al. 2011; Martucci et al. 2012), stationary phases in chromatography (Bruzzoniti et al. 2007; Katiyar et al. 2005; Yasmin and Müller 2011; Zhao et al. 2002) and many others. There is many actual reviews dealing with applications of mesoporous silicas in the literature (Bagheri et al. 2018; Cashin et al. 2018; Croissant et al. 2018; Da'na 2017; Walcarius 2018).

Chemical functionalization is almost always applied to alter the final properties and bring templated mesoporous silica materials closer to a wide range of environmental remediation applications. Two different strategies are usually employed to provide the desired surface modification: co-condensation (Barczak et al. 2013; Yokoi et al. 2003; Zhang et al. 2008) and post-synthesis grafting (Mureseanu et al. 2008; Trindade et al. 2012; Yokoi et al. 2003). Both strategies lead to the materials differing in the reactivity, pore accessibility and distribution of functional moieties, both have also some disadvantages (Barczak 2018). Co-condensation method allows a direct incorporation of functional groups onto the silica surface (and most probably also into mesoporous framework) during one-stage treatment. However, the higher concentrations 
of functional co-monomer(s) cause deterioration of the ordered structure and porosity also due to their destructive effect on surfactant micelles formed prior to the addition of the silica monomers. This is not observed in the case of post-synthesis grafting because functional co-monomer is linked to the hydroxylated surface after completion of the synthesis of mesoporous silica. The main drawback of post-synthesis grafting is that the introduced groups are often unevenly distributed inside the pore walls and external particle surface. Usually density of grafted functional groups is much higher near the pore openings limiting at the same time the access (and-as a consequence-the number) of grafted functionalities inside the mesopores (Kecht et al. 2008).

Many different pendant functional groups has been successfully attached by co-condensation and/or grafting including hydrocarbon groups (e.g., alkyl (Barczak et al. 2009a; Li et al. 2015), vinyl (Barczak et al. 2009b; Wang et al. 2004), phenyl (Barczak et al. 2010b; Fan et al. 2017; Huang et al. 2013)) as well as the groups with complexing properties (amine (Barczak et al. 2013; Chong and Zhao 2003; Dobrowolski et al. 2013; Zhu et al. 2012), thiol (Barczak et al. 2016; Crudden et al. 2005; Liu et al. 2000), pyridine (Barczak 2018), carboxyl (Han et al. 2007; Shen et al. 2008; Tsai et al. 2016)). Co-condensation also allows for incorporation of bridged multi-silylated monomers resulting in the so-called periodic mesoporous organosilicas (Asefa et al. 1999; Inagaki et al. 2002; Mizoshita et al. 2011; Yoshina-Ishii et al. 1999). Those materials have uniformly incorporated organic bridges (instead of pendant groups) covalently bonded to two or more silicon atoms in the silica framework.

Among all functionalized mesoporous organosilicas amine-functionalized ones are most widely studied due to complexing properties of amine groups which renders those materials particularly useful in sorption-based applications where proper porous structure and tailored surface chemistry are two factors tremendously affecting the adsorption processes.

To date amine-functionalized mesoporous materials have been widely tested for removal of heavy metal ions (Aguado et al. 2009; Barczak et al. 2013; Da' na 2017; McManamon et al. 2012), phenols (Toufaily et al. 2013), $\mathrm{H}_{2} \mathrm{~S}$ (Jaiboon et al. 2014), sequestration of carbon dioxide (Chang et al. 2009; Gunathilake et al. 2016) but their use for removal of hazardous pharmaceuticals from aqueous media is barely explored (Barczak et al. 2018). Amine groups are usually incorporated by the use of aminopropyltriethoxysilane (APTES) or aminopropyltrimethoxysilane (APTMS) either by co-condensation or post-synthesis grafting. Very often, when higher concentration of amine groups is needed other monomers containing two or three amine groups can be used, namely $N$-[3-(trimethoxysilyl)propyl]-ethylenediamine
(TMPED) or (3-trimethoxysilylpropyl)diethylenetriamine (TMPET), having two and three amine groups, respectively.

Regardless of the type of aminosilane, there is a serious problem associated with their incorporation by cocondensation already mentioned before, i.e., destruction of the ordered mesophase resulting in reduced porosity and ordering. The destructive effect of amine groups can be partially overcome by increasing the time interval between addition of silica monomer (usually tetraethoxysilane) and amine-functional monomer (usually aminopropyltrialkoxysilane). This maneuver allows for the creation of initial silica framework robust enough against destructive action of the second monomer. The drawback of this approach is the reduced functionalization efficiency, i.e., only a part of amine groups is successfully incorporated within the silica framework with simultaneous deterioration of the ordering and porosity (Barczak et al. 2018). In this paper the possible solution to the-above described problem is investigated, i.e., addition of the bis-silylated silica monomer prior to addition of aminosilane. This approach can be used to tune the structural, chemical but also sorption-related properties which has been shown here.

\section{Experimental}

\subsection{Reagents}

The following reagents were used as received: triblock copolymer Pluronic P123 (P123, Mn $=5800$, SigmaAldrich), tetraethoxysilane (TEOS, 99\%, Sigma-Aldrich), 3-trimethoxysilylpropyldiethylenetriamine (TMPET, 95\%, Fluorochem), 1,2-bis(triethoxysilyl)ethane 95\% (BTESE, 96\%, Sigma-Aldrich), 1,4-bis(triethoxysilyl)benzene 96\% (BTESB, 96\%, Sigma-Aldrich), 4,4-Bis(trimethoxysilyl)1,1-biphenyl (BTMSD, 95\%, Sigma-Aldrich), $\mathrm{HCl}$ (36\%, $\mathrm{POCH}), \mathrm{NaOH}(\mathrm{POCH}), \mathrm{NaCl}(\mathrm{POCH})$, ethanol (EtOH, 99.8\%, POCH), (DICL, > 98\%, Sigma-Aldrich).

\subsection{Synthesis of the xerogels}

The synthesis of the samples was adopted from our previous papers (Barczak et al. 2018, 2010a, 2009b). $2 \mathrm{~g}$ of P123 was dissolved in $72 \mathrm{~mL}$ of $1.75 \mathrm{M} \mathrm{HCl}$ under stirring at $40{ }^{\circ} \mathrm{C}$. After $8 \mathrm{~h}$ of stirring $14 \mathrm{mmol}$ of TEOS was added dropwise to this solution. After $15 \mathrm{~min} 2 \mathrm{mmol}$ bridged monomer was added (BTESE-sample R2, BTESB-sample R3 or BTMSD—sample R4) followed by addition of $2 \mathrm{mmol}$ of TMPET after next $15 \mathrm{~min}$. For the reference purposes sample without bridged monomer, i.e., R1, was also synthesized by addition of $2 \mathrm{mmol}$ of TMPET to $18 \mathrm{mmol}$ of TEOS with interval time $30 \mathrm{~min}$. The resulting mixtures were stirred at $40{ }^{\circ} \mathrm{C}$ for $24 \mathrm{~h}$ followed by ageing at $100{ }^{\circ} \mathrm{C}$ for the next 
$24 \mathrm{~h}$. The precipitated solids were thoroughly washed with distilled water, filtered and extracted three times with acidified absolute ethanol (each portion was composed of $98 \mathrm{~mL}$ of $96 \%$ ethanol and $2 \mathrm{~mL}$ of conc. $\mathrm{HCl}$ ) at $80{ }^{\circ} \mathrm{C}$ for $4 \mathrm{~h}$ to remove the template. After filtration, the obtained powders were again thoroughly washed with distilled water, filtered again and dried overnight at $80{ }^{\circ} \mathrm{C}$.

\subsection{Instrumental characterization}

The nitrogen isotherms were measured at $-196{ }^{\circ} \mathrm{C}$ using an Quantachrome 1200 e analyzer after degassing the samples at $110{ }^{\circ} \mathrm{C}$ in vacuum for $12 \mathrm{~h}$. The BET specific surface areas $\left(\mathrm{S}_{\mathrm{BET}}\right)$ were evaluated in the range of relative pressures $\mathrm{p} /$ $\mathrm{p}_{0}$ of $0.05-0.20$. The total pore volumes $\left(\mathrm{V}_{\text {total }}\right)$ were calculated by converting the amount adsorbed at $\mathrm{p} / \mathrm{p}_{0}=0.99$ to the volume of liquid adsorbate. Micropore pore volume $\left(\mathrm{V}_{\text {micro }}\right)$ were calculated using Saito-Foley method (Saito and Foley 1991). The average pore sizes $\left(\mathrm{d}_{\mathrm{DFT}}\right)$ were estimated using the NLDFT method using ASiQwin 3.0 software (Quantachrome). The CHN elemental analysis was carried out using the Perkin Elmer CHN 2400 analyzer. The FTIR spectra were measured by means of FTIR 6200 spectrometer (Jasco) in the range of $4000-400 \mathrm{~cm}^{-1}$ with the resolution $4 \mathrm{~cm}^{-1}$ by averaging 32 scans. The TEM imaging of randomly selected parts of the surface was performed on Tecnai G20 X-Twin (FEI) microscope with $200 \mathrm{keV}$ accelerating voltage. The SEM imaging of randomly selected parts of the surface was performed under high vacuum conditions by means of Quanta 3DFEG (FEI) microscope with the accelerating voltage 5-30 keV. The samples were coated with Pd/Au using the Polaron SC7640/CA7625 (Quorum Technologies) sputter coater. The zeta potential was evaluated using Zetasizer Nano ZS (Malvern Instruments). Suspensions were prepared by dispersing $\sim 0.02 \mathrm{~g}$ of ground samples in $4 \mathrm{~mL}$ of $1 \times 10^{-3} \mathrm{M} \mathrm{KCl}$. Thermal analyses (TG) were carried out in oxygen atmosphere on a STA 449 Jupiter F1 (Netzsch, Germany) with sensor thermocouple type S TG-DTA under the following operational conditions: heating rate $10{ }^{\circ} \mathrm{C} \mathrm{min}{ }^{-1}$, oxygen flow: $50 \mathrm{~mL} \mathrm{~min}{ }^{-1}$, temp. range: $25-600{ }^{\circ} \mathrm{C}$, sample mass: $\sim 5 \mathrm{mg}$. Diffraction patterns were measured using Empyrean X-ray diffractometer (PANalytical, The Netherlands) equipped with copper anode and multilayer focusing mirrors. Samples were measured in $\theta-2 \theta$ geometry over a range from 0.2 to $7^{\circ}$ with step size of $0.012^{\circ}$. The beam slit was $1 / 16 \mathrm{~mm}$.

\subsection{Sorption of pharmaceuticals}

In each experiment $\sim 10 \mathrm{mg}$ of adsorbent was shaken for $16 \mathrm{~h}$ with $30 \mathrm{~mL}$ solution of different DICL aliquots (namely $25,50,100,150,250,500$ and $1000 \mathrm{mg} \mathrm{L}^{-1}$ ). The $\mathrm{pH}$ was adjusted to 5.5 using $10^{-3} \mathrm{M} \mathrm{NaOH}$ and $10^{-3} \mathrm{M} \mathrm{HCl}$. The equilibrium adsorption amounts were calculated from mass balance using the formula: $a=\left(c_{i}-c_{j}\right) V^{-1}$, where $c_{i}$ is the initial concentration $\left(\mathrm{mg} \mathrm{L}^{-1}\right), \mathrm{c}_{\mathrm{j}}$ is the equilibrium concentration $\left(\mathrm{mg} \mathrm{L}^{-1}\right), \mathrm{V}$ is the volume of the solution (L) and $\mathrm{m}$ is the mass of the adsorbent $(\mathrm{g})$. Measurements of DICL concentrations were completed using the UV-Vis spectrometer Specord 200 (Analytic Jena) at wavelength $278 \mathrm{~nm}$. The small volume of the solution was filtered before measurement using syringe $0.45 \mu \mathrm{m}$ filter.

\subsection{DFT calculations}

Calculations of equilibrium geometries and harmonic vibrational frequencies of the selected representative fragments were carried out at the DFT/B3LYP computational level with (Becke 1993) 6-311++G** basis set (Frisch et al. 1984; Krishnan et al. 1980). All optimized structures turned out to be local minima (i.e., all vibrational frequencies were real numbers). Calculations were performed using the PQS quantum chemistry package (Baker et al. 2009). The relative energies for the process $\mathrm{A}+\mathrm{B}+\ldots \rightarrow \mathrm{C}+\mathrm{D}+\ldots$ were calculated according to the following formula: $E_{\mathrm{C}}+E_{\mathrm{D}}+\ldots-E_{\mathrm{A}}-E_{\mathrm{B}} \ldots$ The negative value indicates that products are more stable than substrates.

\section{Results}

The names of the samples along with physico-chemical characterization are presented in Table 1 and the scheme of the synthesis-in Fig. 1. The molar ratio 14:2:2 (sample R2-R4) or 18:2 (sample R1) was chosen to keep constant number of silicon atoms in each reaction mixture (note that one mmol of bis-silylated bridged monomer, i.e., BTESE, BTESB, BTMSD contains $2 \mathrm{mmol}$ of Si atoms). As follows from our previous investigations the addition of $10 \%$ of TMPET results in a significant decrease of porosity from the one hand but very well DICL sorption uptakes from the other (Barczak et al. 2018) thus it was decided to keep that ratio here. It was suggested in the literature that the addition of bis-silylated bridged monomer can oppose destructive action of amino silane (Barczak et al. 2009b). Indeed looking at the adsorption isotherms presented in Fig. 2a it can be seen that samples synthesized with the addition of BTESE (sample R2), BTESB (sample R3) or BTMSD (sample R4) have more developed porosity than the sample R1. In the case of the sample R3 the shape of isotherm with H1 type of the hysteresis loop is typical for highly ordered SBA-15 mesoporous structure. The remaining samples exhibit $\mathrm{H} 2 / \mathrm{H} 3$ type of hysteresis loop indicating rather disordered mesoporous structure. Pore size distributions presented in Fig. $2 b$ have two maxima: one located in the range $3.3-4.0 \mathrm{~nm}$ and the other located in the range $8.5-11.7 \mathrm{~nm}$. 


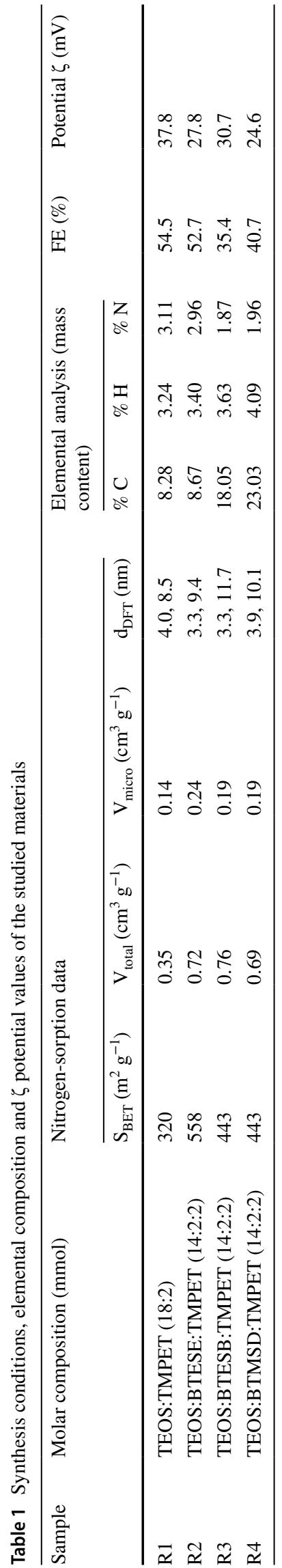

The second maximum is due to the primary mesopores created due to the removal of the template (Pluronic P123). In Table 1 the values of porous structure parameters are shown. Addition of the bis-silylated bridged monomer increase the average size of primary mesopore which is particularly visible for the sample R3 (11.7 $\mathrm{nm})$. Sample R2 has the highest value of specific surface area $\left(\mathrm{S}_{\mathrm{BET}}\right)$ and micropore volume $\left(\mathrm{V}_{\text {micro }}\right)$ among all the samples studied. Interestingly total pore volumes $\left(\mathrm{V}_{\text {total }}\right)$ of the samples $\mathrm{R} 2-\mathrm{R} 4$ are two times higher than that of the sample R1. All the observed changes testify that addition of bis-silylated bridged monomer remarkably influences the porous structure of the final samples.

As can be seen from the low-angle XRD patterns (Fig. 3) all the materials exhibit an ordering, albeit with different degree and type. In the case of the sample R3 there are three well resolved peaks in the range of $2 \theta=0.8-1.8^{\circ}$, one sharp reflection centered at $2 \theta=0.8^{\circ}$ indexed as (100), and two minor but distinct reflections at $2 \theta=1.5^{\circ}$ and $2 \theta=1.7^{\circ}$, indexed as (110) and (200), respectively. This pattern, related to the hexagonal $p 6 \mathrm{~mm}$ symmetry, is characteristic of well-developed SBA-15 mesostructure (Barczak et al. 2010a, 2009b). In the case of the sample R1 there are two reflections related to the cubic Ia3d symmetry: a well resolved one at $0.9^{\circ}$ and a smaller hump at $1.1^{\circ}$ indexed as (211) and (220) respectively (Barczak et al. 2009b; Liu et al. 2002). Decreased peak intensity of the (220) reflection can be related to the presence of organic moieties (coming from the co-condensed TMPET monomer) and inside the pores which could false diffract (Visuvamithiran et al. 2013). In the case of the samples R2 and R4 it is difficult to clearly assign a specific space group because there is only one reflection centered at $2 \theta=1.8^{\circ}$. This also means that the ordering of the samples R2 and R4 is deteriorated, most probably due to presence of specific co-monomers in the initial mixture adversely affecting the formation of the mesophase during the synthesis.

Interestingly the addition of BTESB bridged monomer preserve the $p 6 \mathrm{~mm}$ symmetry of the final R3 sample, in contrast to remaining bridged monomers. Another interesting observation is that the specific combination of monomers can induce a change of the symmetry from hexagonal to cubic, despite the fact that the synthesis conditions were set to obtain samples with hexagonal symmetry. We reported previously that the presence of vinyltriethoxysilane co-monomer can result in cubic symmetry (Barczak et al. 2009b); from this research it follows that also TMPET behaves similarly. Previously it was observed for 3-mercaptopropyltriethoxysilane (Garcia-Bennet et al. 2004). Most probably this is a more common feature of the hybrid silica structure synthesized by co-condensation of pure silica monomer (like TEOS) and organic monomer with pendant group (like TMPET) and is governed by the amount of co-monomer and 

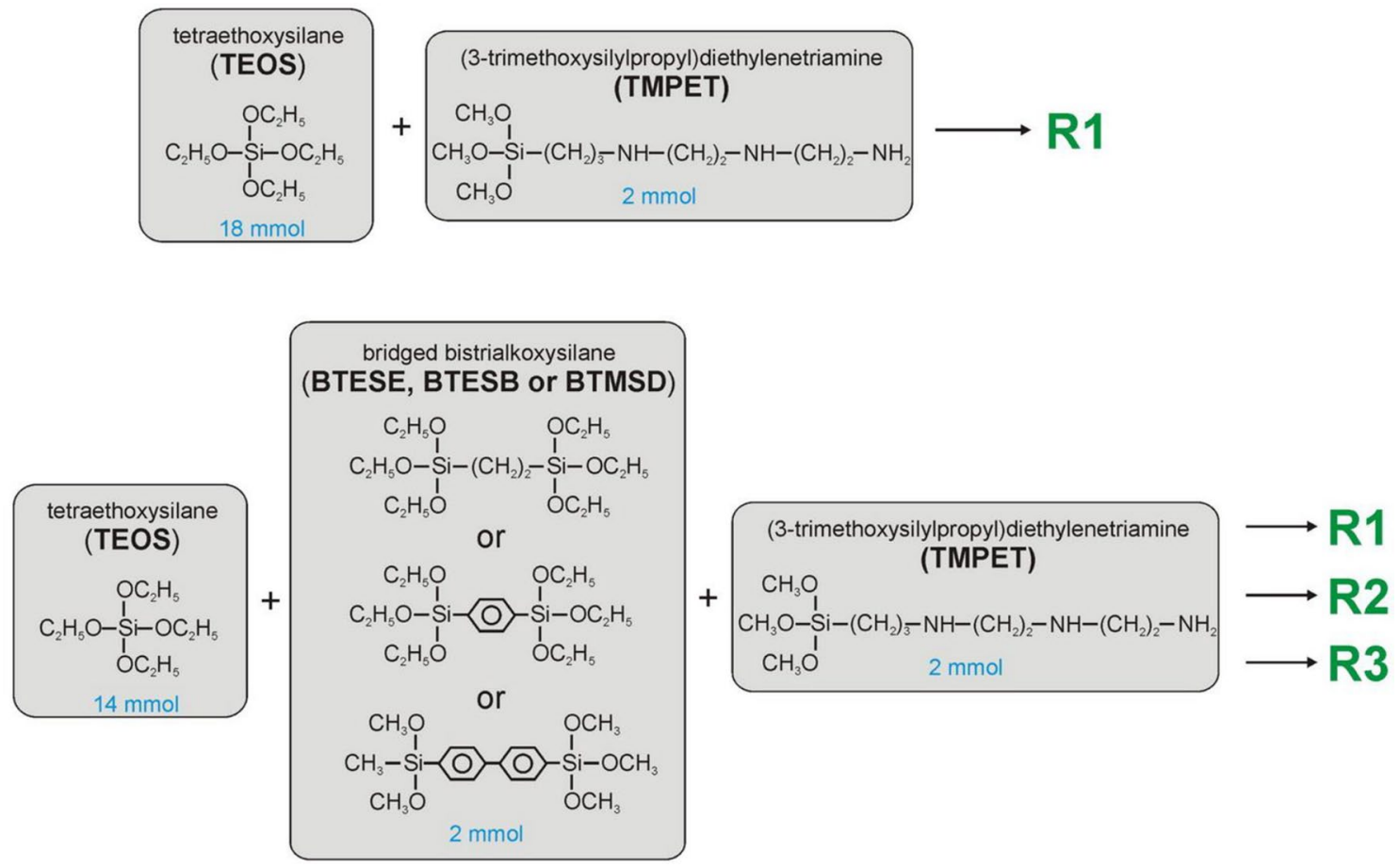

Fig. 1 Scheme of co-condensation leading to the amine-functionalized silica materials
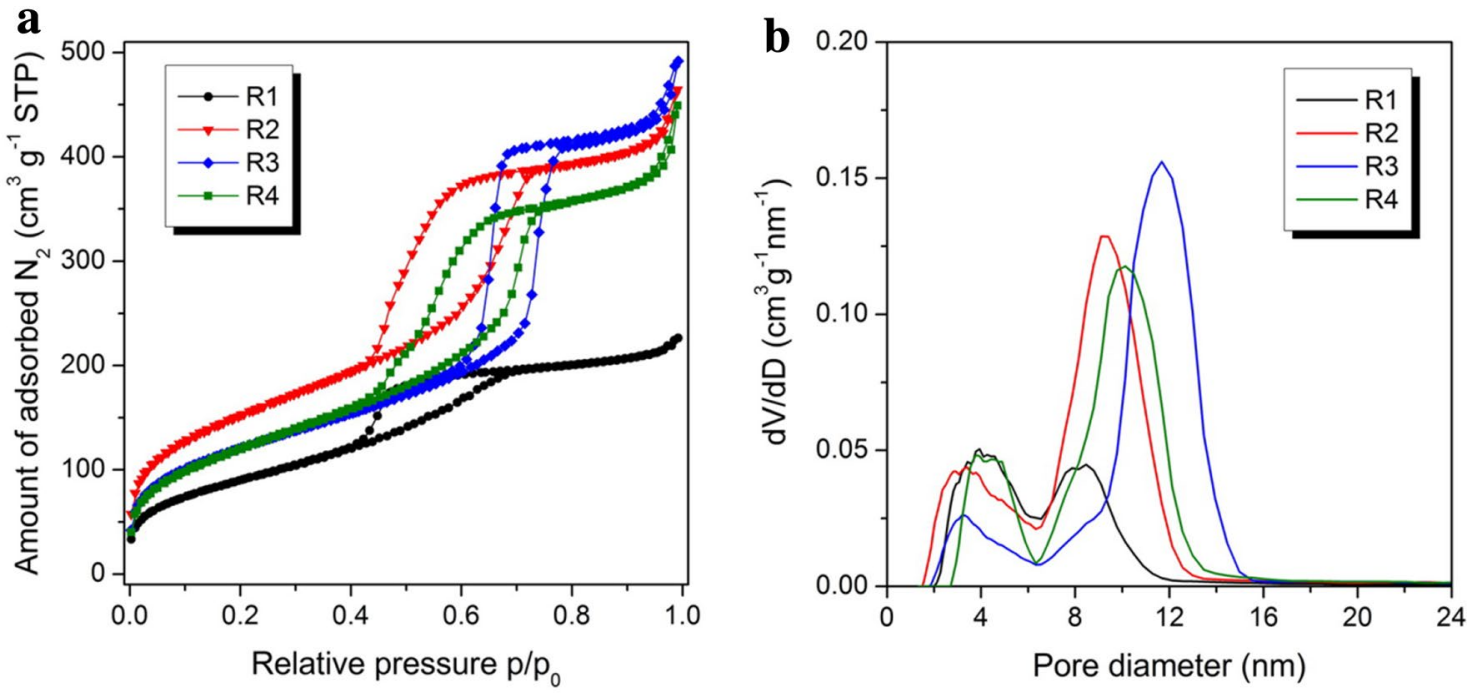

Fig. 2 Adsorption isotherms (a) and the corresponding pore size distributions (b) of the samples studied

most probably other synthesis parameter (e.g., processing temperature). The detailed explanation of this phenomenon exceeds the scope of this paper.

The contents of nitrogen, carbon and hydrogen are given in Table 1. Different nitrogen contents indicate successful co-condensation, albeit with different functionalization efficiencies (FE, calculated as a ratio of the measured and theoretical content of nitrogen). While the addition of BTESE does not change significantly the FE the same is not true for the BTESB and BTMSD—samples R3 and R4 have significantly lower nitrogen contents and, consequently, lower FE values. The common feature of those two above-mentioned monomers is that they possess the benzene rings thus some additional interactions between the rings and amine groups can be responsible for the resulting lower functionalization efficiency. Thus 


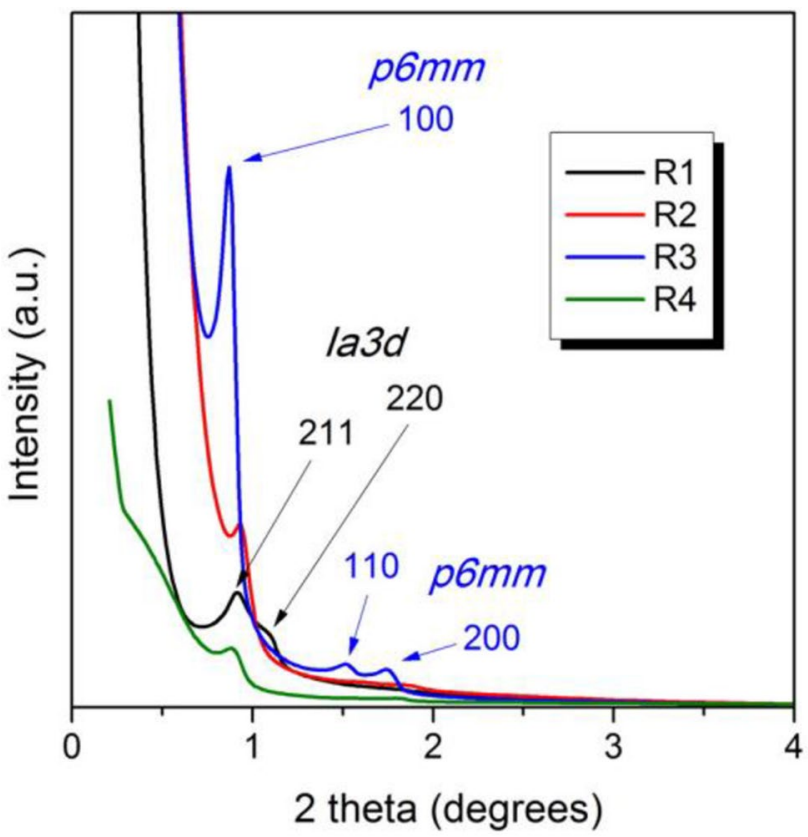

Fig. 3 Low-angle X-ray diffractograms of the samples studied

TMPET molecules can be specifically oriented (in a statistical sense) during the synthesis which may result in their limited hydrolysis and co-condensation.

To further investigate the chemistry of the studied samples FTIR spectra were collected (Fig. 4). The most intensive band with the maximum at $\sim 1075 \mathrm{~cm}^{-1}$ which has a shoulder at $1150-1250 \mathrm{~cm}^{-1}$ can be attributed to stretching modes of the siloxane framework. The bands located at $\sim 950 \mathrm{~cm}^{-1}, \sim 800 \mathrm{~cm}^{-1}$ and $\sim 450 \mathrm{~cm}^{-1}$ can be assigned to $\mathrm{Si}-\mathrm{O}$ vibrations in the $\mathrm{Si}-\mathrm{OH}$ moieties, symmetric stretching vibrations of $\mathrm{Si}-\mathrm{O}-\mathrm{Si}$, and bending vibrations of $\mathrm{Si}-\mathrm{O}-\mathrm{Si}$, respectively (Sanaeishoar et al. 2015).

The presence of the $-\mathrm{CH}_{2}-$ link in the alkyl chain of TMPET and ethylene bridge of BTESE is confirmed by a group of absorption bands in the region of $\sim 2800-3000 \mathrm{~cm}^{-1}$, attributed to the stretching modes of $\mathrm{CH}_{2}$ groups, and possibly $\mathrm{CH}_{3}$ groups (fragments of residual ethoxy groups as well us unremoved traces of template). The higher intensity of those signals for the samples R3 and R4 may testify to considerable amount of unremoved Pluronic P123. Two absorption bands of stretching vibrations of amine groups located in the region of $3300-3500 \mathrm{~cm}^{-1}$ could not be detected due to the presence of a wide band of the physically adsorbed water extending from $\sim 3700 \mathrm{~cm}^{-1}$ to $\sim 3000 \mathrm{~cm}^{-1}$. In the case of the samples R3 and R4 the presence of benzene rings should be manifested by not very intense bands in the range of 3000-3100, ca. 1600 and ca. $1500 \mathrm{~cm}^{-1}$ although due to low concentrations of the other co-monomers co-condensed with TEOS there are no clear signals on the FTIR spectra.
Thermal stability of the synthesized samples was evaluated by means of thermal gravimetry (Fig. 5). Sample R1 reveals a total mass loss of approximately $20 \%$, due to the three distinctive but often overlapping processes occurring during thermal treatment: the evaporation of physically adsorbed water $\left(<150{ }^{\circ} \mathrm{C}, \sim 4 \%\right.$ mass loss $)$ decomposition of organic fragments $\left(150-450{ }^{\circ} \mathrm{C}, \sim 14 \%\right.$ mass loss) and dehydroxylation of surface silanols $\left(>450{ }^{\circ} \mathrm{C}, \sim 2 \%\right.$ mass loss). Two distinct DTG maxima observed at $75{ }^{\circ} \mathrm{C}$ and $290{ }^{\circ} \mathrm{C}$ correspond to the first two processes. Interestingly, the location of those maxima vary from sample to sample. The first maximum is centered at $70-75^{\circ} \mathrm{C}$ for the samples $\mathrm{R} 1, \mathrm{R} 3$ and $\mathrm{R} 4$ but it is shifted to $85^{\circ} \mathrm{C}$ for the sample $\mathrm{R} 2$. This means that water is bound stronger in the pores of $\mathrm{R} 2$ sample than in the case of the rest of the samples. This may be due to the highest content of micropores (cf. Table 2) where a significant part of water can be stored. The second maximum on DTG curves is centered at $\sim 285{ }^{\circ} \mathrm{C}$ for the samples R1 and R2 and at $\sim 360{ }^{\circ} \mathrm{C}$ for the samples R3 and $\mathrm{R} 4$. This maximum arises from the decomposition of pendant propyldiethylenetriamine groups as well as the organic bridges. In the case of R3 and R4 samples, this maximum is shifted towards higher temperatures which means that the benzene bridges make the silica samples more resistant to thermal decomposition. In the case of the samples R3 and particularly R4 the third maximum arises at $600^{\circ} \mathrm{C}$ due to the remarkable dehydroxylation of the surface silanols. The results of TG and DTG analyses clearly show that cocondensation of the monomers was successfully achieved.

The SEM and TEM data presented in Figs. 6 and 7, respectively show that the morphology is typical for SBA15 , i.e., it is composed of the "sausage-like" hexagonal motifs often curved and connected to others along long edges. The TEM microphotographs reveal that those motifs are formed by the ordered mesoporous network of long uniform hexagonal mesopores with the diameter of several nm, which is consistent with the pore sizes obtained from the nitrogen sorption data (cf. Table 1).

To reveal possible application of the fabricated xerogels for removal of pharmaceuticals as well as to correlate their physico-chemical properties with sorption efficiencies, adsorption of diclofenac sodium salt (DICL) was studied. Diclofenac poses a significant risk to the environment and to human health thus it is considered as one of the priority pollutants in the European Water Framework Directive. DICL is a commonly prescribed in large quantities, and-as a result-it has being detected in wastewaters along with its metabolites and products of its photo-transformational changes. Chronic exposure to DICL has adverse effects for aquatic animals and humans (Barczak et al. 2018).

From our previous works it follows that the adsorption of DICL is very fast and usually $2-4 \mathrm{~h}$ are needed to reach equilibrium and the pH 6 is optimal (Barczak 2018; Barczak 

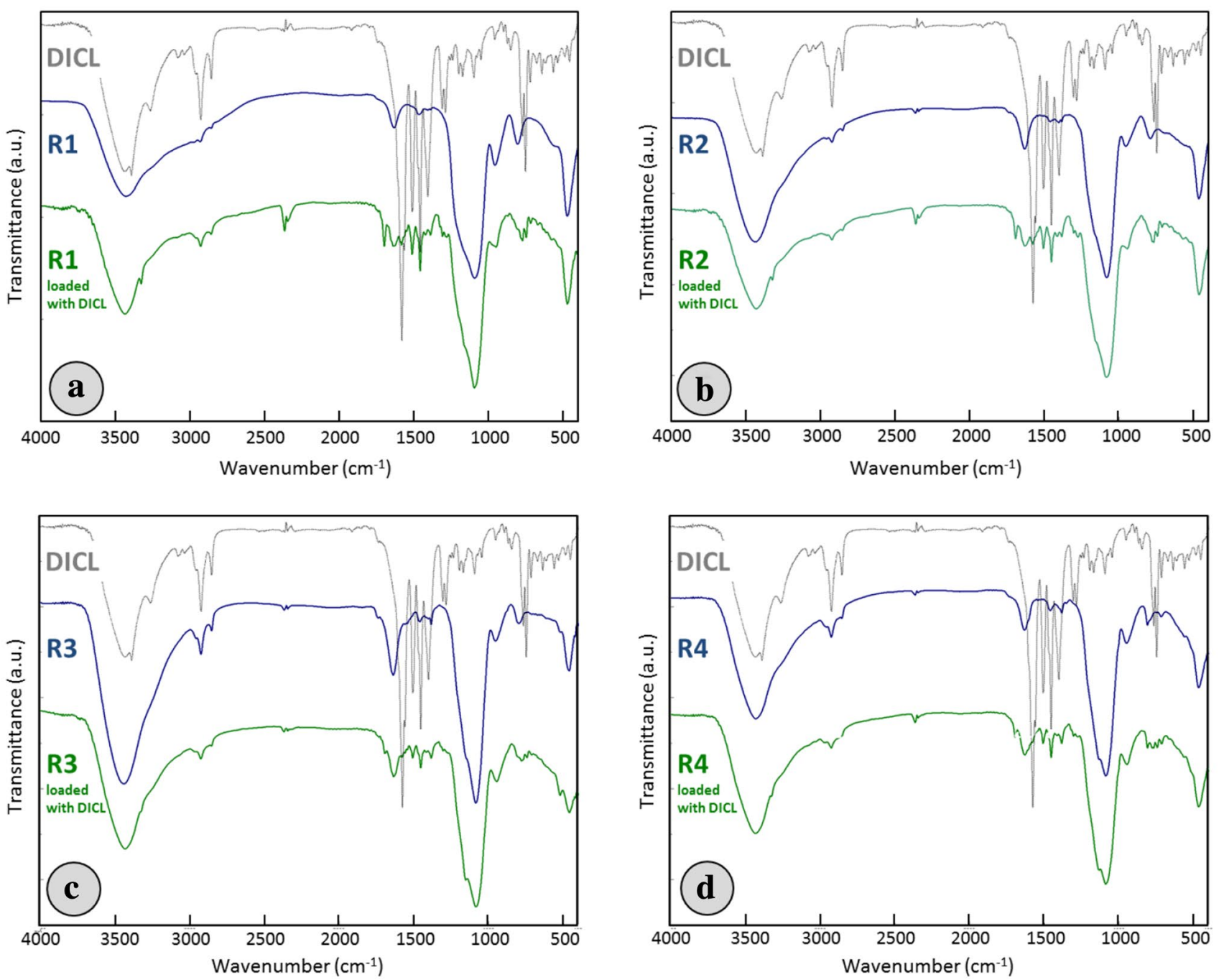

Fig. 4 FTIR spectra of the samples R1-R4 (a-d) studied before and after sorption of DICL (spectrum of DICL was added to each graph for better comparison)

et al. 2018). Thus the sorption experiments were run for $16 \mathrm{~h}$ at $\mathrm{pH} 6$ (unbuffered solution). The observed static sorption capacities (SSC) are given in Table 2. As it can be seen, samples R1 and R2 exhibit highest SSC values of 841.8 and $808.7 \mathrm{mg} \mathrm{g}^{-1}$, respectively, while the samples R3 and R4 have lower SSC values of 607.6 and $394.5 \mathrm{mg} \mathrm{g}^{-1}$, respectively. The higher SSC values for R1 and R2 are obviously related to higher number of protonated amine groups ready to electrostatically interact with DICL anions. In the case of samples R3 and R4, the lower number of amine groups results in lower SSC values. However, despite the similar nitrogen content (R3: $1.87 \%$ vs. R4: 1.96\%) there is a huge difference in SSC values. Both samples have similar porous structure so the reason of this behaviour must by related more to the surface chemistry than porosity. Looking at the values of potential $\zeta$ collected in Table 1, it can be seen that all the samples have positive values of $\zeta$ which means that the surface of all the sorbents can electrostatically attract DICL anions (pKa value of DICF which is 4.15 (Barczak et al. 2018) thus at pH 6 the soluble ionized form of DICF is almost exclusively present in the solution). Among all the samples, R4 has the lowest value of $\zeta$ (24.6) which means that the electrostatic interactions are the most suppressed for this adsorption system. The highest mass loss $>450{ }^{\circ} \mathrm{C}$ observed on the TG curve of this sample (Fig. 5a) due to the dehydroxylation testifies to the abundance of silanol group present on the surface (reflected in lower $\zeta$ value). Thus the resulting charge density is not only controlled by the amine groups but also by the silanol groups, whose are mostly deprotonated at $\mathrm{pH} 6$. Thus, even for surface-functionalized mesoporous silica, the effective charge density is not only controlled by the incorporated functionalities but also by the number and form (protonated vs. deprotonated) of the silanol groups (Rosenholm et al. 2007). 
Fig. 5 The TG (a) and DTG (b) curves in oxygen for the samples R1-R4

Table 2 Fitting parameters for the studied adsorption systems

Fig. 6 SEM images of the selected samples (R1-a, b; $\mathrm{R} 4-\mathbf{c}, \mathbf{d})$
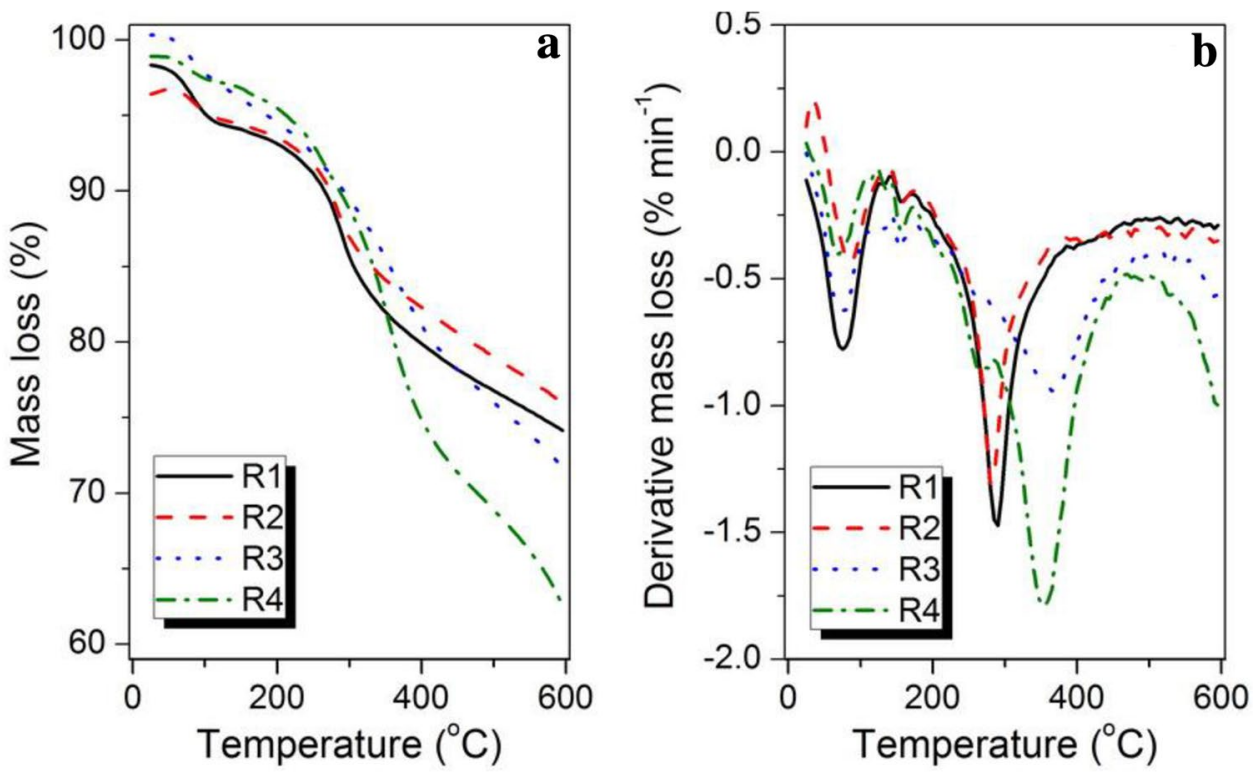
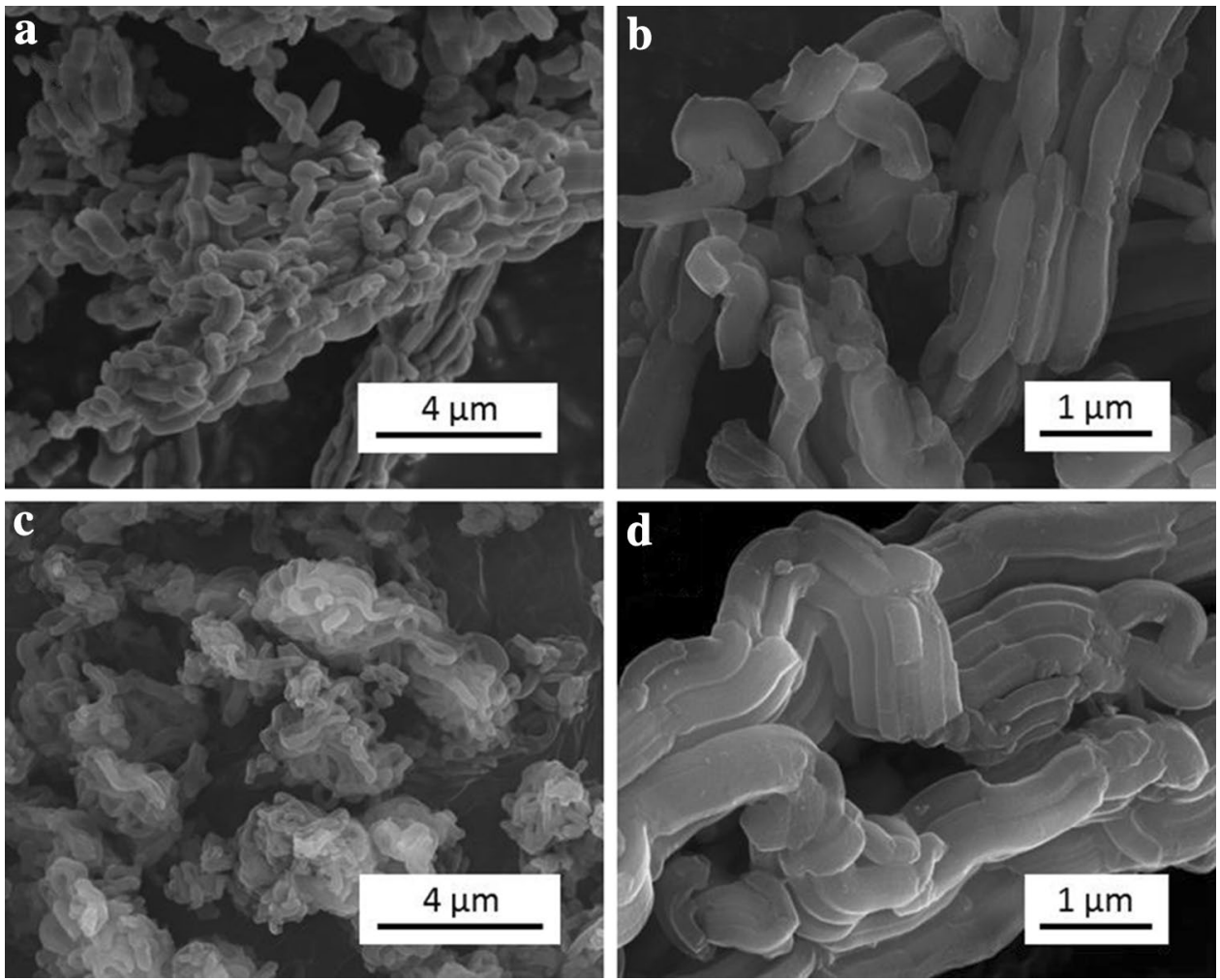

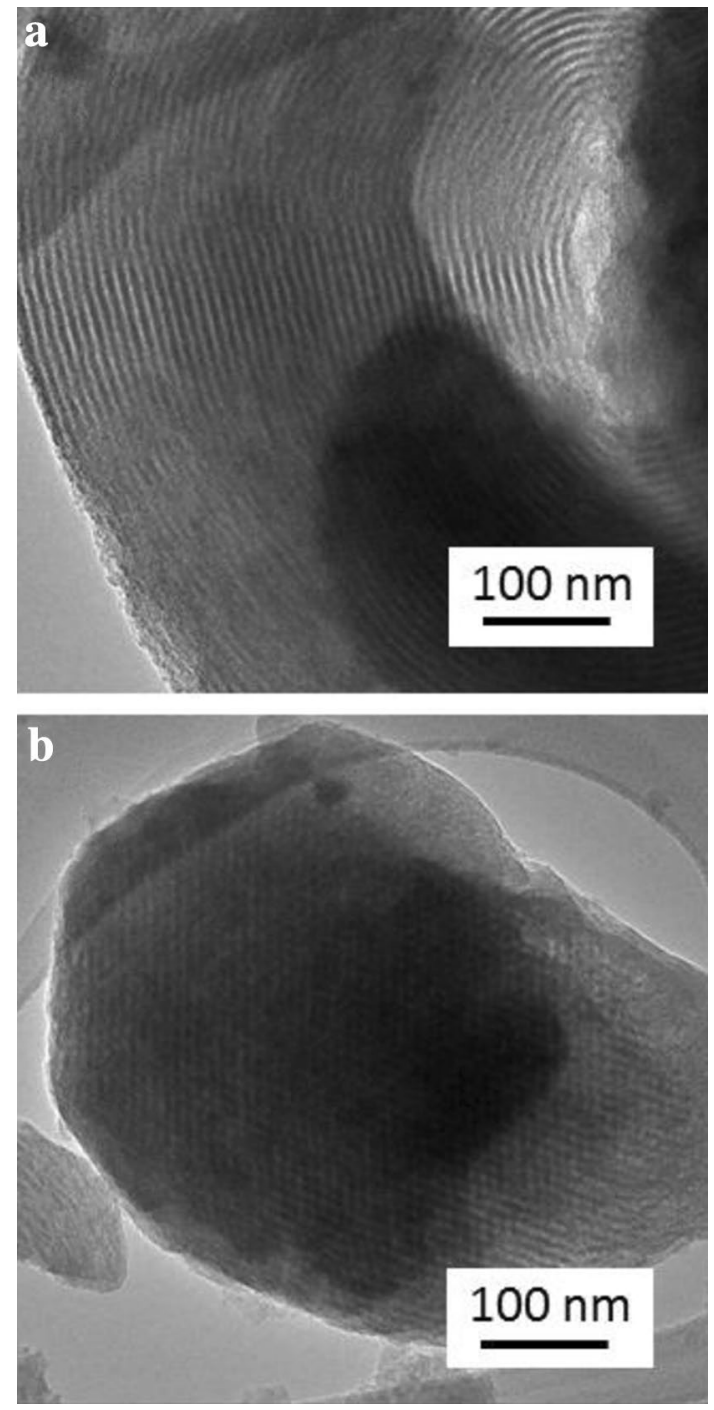

Fig. 7 TEM images of the sample R1

The adsorption isotherms are presented in Fig. 8 along with their fitting using three common adsorption models: Langmuir, Freundlich and Sips (also called Langmuir-Freundlich). The proper formulas can be found in one of our previous works (Barczak 2018; Barczak et al. 2018). Fitting parameters are given in Table 2 . The correlation coefficients show that Sips and Langmuir models fit the results better than the Freundlich model. However, correlation coefficients are noticeably better for Sips than for Langmuir model. Interestingly the $\mathrm{K}_{\mathrm{L}}$ and $\mathrm{K}_{\mathrm{LF}}$ constants, (also called affinity constants) are significantly higher for R4 sample when compared with the rest of the samples. It should be mentioned that the fitting results should be interpreted cautiously since Sips equation has three parameter so it can fit per se the isotherms better that two-parameter models of Langmuir and Freundlich.
The presence of adsorbed DICL is unquestionably confirmed by FTIR spectroscopy-spectra of loaded sorbents are presented in Fig. 3 (for convenience, the spectrum of DICL sodium salt is also given at each spectrum). Comparing the spectra of the samples before and after adsorption it can be seen that there are additional signals characteristic of DICL, namely: $3322 \mathrm{~cm}^{-1}$ (amine stretching), $1560 \mathrm{~cm}^{-1}$ (aromatic symmetric CC stretching), 1506 and $1453 \mathrm{~cm}^{-1}$ (asymmetric ring stretching and symmetric deformation of methylene groups, respectively), 1576 and $1381 \mathrm{~cm}^{-1}$ (stretching modes of carboxylate group), 1302 and $1279 \mathrm{~cm}^{-1}$ (C-N stretching).

In this work regeneration was achieved by immersing the loaded sorbents in $0.9 \% \mathrm{NaCl}$ at $40{ }^{\circ} \mathrm{C}$. Full desorption was achieved after $48 \mathrm{~h}$ which was confirmed by FTIR spectra of the samples after desorption exhibiting lack of signals characteristic of DICL. Desorption curves aligned as releasing profiles are shown in Fig. 9. Although all the profiles look similar there is a very important difference between them: amount of desorbed DICL after only one minute of contact with $\mathrm{NaCl}$ solution vary significantly from sample to sample: for R1 $=30 \%$ of DICL is immediately released, for R2: $\sim 20 \%$, while for R3 and R4 only $7 \%$ and $2 \%$, respectively. Obviously, the immersion of sorbents in $0.9 \% \mathrm{NaCl}$ solution (ionic strength: $154 \mathrm{mmol}$ ) suppresses the electrostatic interactions between the silica surface and deprotonated DICL anions which results in immediate release of some portion of the drug. This means that the significant amount of DICL is physically bounded to the surface via electrostatic interactions. It was already suggested and confirmed by both experimental findings and theoretical calculations that the first layer of adsorbed DICL is strongly bonded by hydrogen bonds formation between protonated amine groups and carboxylate anions but the next layers of the adsorbed DICL are due to the electrostatic Coulombian interactions (Barczak et al. 2018). Those findings are in agreement with the current state-of-the-art where above-mentioned mechanisms along with hydrophobic interactions are usually considered as three possible mechanisms responsible for the diclofenac adsorption.

In our previous paper we have shown that the main driving adsorption force between protonated amine groups and diclofenac anion is the hydrogen bond formation (this applies to DICL anions layers having direct access to the protonated amine groups, the remaining anions interact via electrostatic attraction). Thus it was interesting to investigate whether the organic bridges affect the stabilization of the final complex. To verify this the DFT calculations for the $\mathrm{R} 2$ and R3 sorbents were performed in a fashion described in our previous papers (Barczak et al. 2018), i.e., we estimated energetic effect associated with the adsorption of $\mathrm{DICL}^{\ominus}$ on the protonated amine groups. For simplicity, we used here the aminopropyl group endcapped with hydrogen 
Fig. 8 Adsorption isotherms and their fitting with Langmuir, Freundlich and Sips (LangmuirFreundlich) models

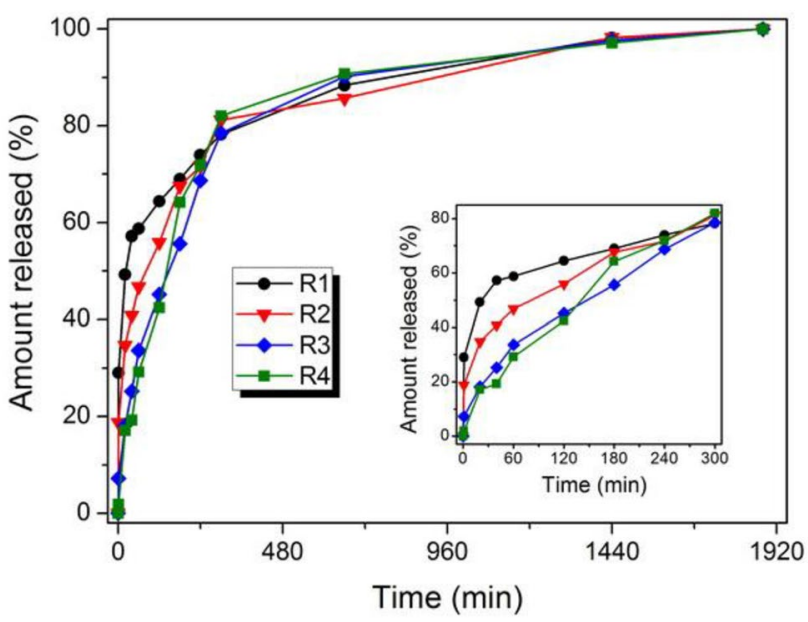

Fig. 9 DICL releasing profiles for the studied samples
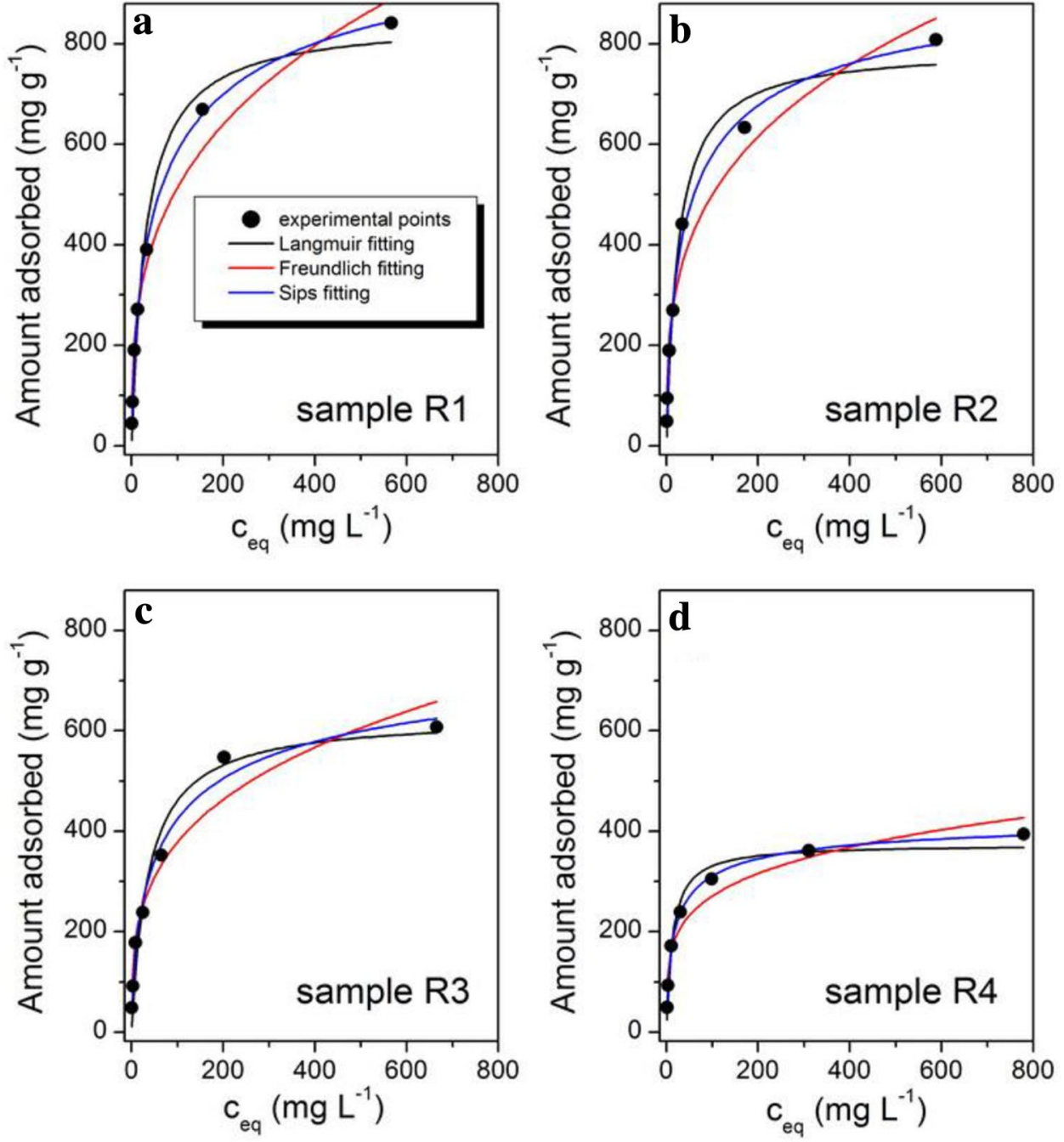

atom, i.e., $\mathrm{CH}_{3}-\left(\mathrm{CH}_{2}\right)_{2}-\mathrm{NH}_{2}$ as in ref. (Barczak et al. 2018) instead of using $\mathrm{CH}_{3}-\left(\mathrm{CH}_{2}\right)_{2}-\mathrm{NH}-\left(\mathrm{CH}_{2}\right)_{2}-\mathrm{NH}-\left(\mathrm{CH}_{2}\right)_{2}-\mathrm{NH}_{2}$ group. This time the protonated $\ldots \mathrm{N}^{\oplus} \mathrm{H}_{3} \cdots \mathrm{H}_{2} \mathrm{O}$ group was allowed to interact with $(\mathrm{MeO})_{3} \mathrm{Si}-\left(\mathrm{CH}_{2}\right)_{2}-\mathrm{Si}(\mathrm{OMe})_{3}$ and $(\mathrm{MeO})_{3} \mathrm{Si}-\mathrm{Ar}-\mathrm{Si}(\mathrm{OMe})_{3}$ systems (note that further simplification consisted in replacement of the ethoxy group with methoxy group was introduced in order to further reduce the system size). It was also assumed that the ... $\mathrm{N}^{\oplus} \mathrm{H}_{3} \cdots \mathrm{H}_{2} \mathrm{O}$ group is a member of a neighboring silica chain (or other structure present in the bulk phase). The structures are shown in Fig. 10. Note that additional hydrogen bond(s) are formed between $\ldots \mathrm{N}^{\oplus} \mathrm{H}_{3} \cdots \mathrm{H}_{2} \mathrm{O}$ and methoxy groups. The calculations in which the $\mathrm{DICL}^{\ominus}$ replaces water molecule clearly show that there is hardly a difference between the stabilization energies, being slightly lower than $-80 \mathrm{kcal} \mathrm{mol}^{-1}$, when $(\mathrm{MeO})_{3} \mathrm{Si}-\left(\mathrm{CH}_{2}\right)_{2}-\mathrm{Si}(\mathrm{OMe})_{3}$ and $(\mathrm{MeO})_{3} \mathrm{Si}-\mathrm{Ar}-\mathrm{Si}(\mathrm{OMe})_{3}$ systems are considered. In addition, the presence of bridges lowers down the stabilization by ca. $20 \mathrm{kcal} \mathrm{mol}^{-1}$, as in the case considered in our previous work the energetic effect is $-103.2 \mathrm{kcal} \mathrm{mol}^{-1}$ (cf. Fig. 8 


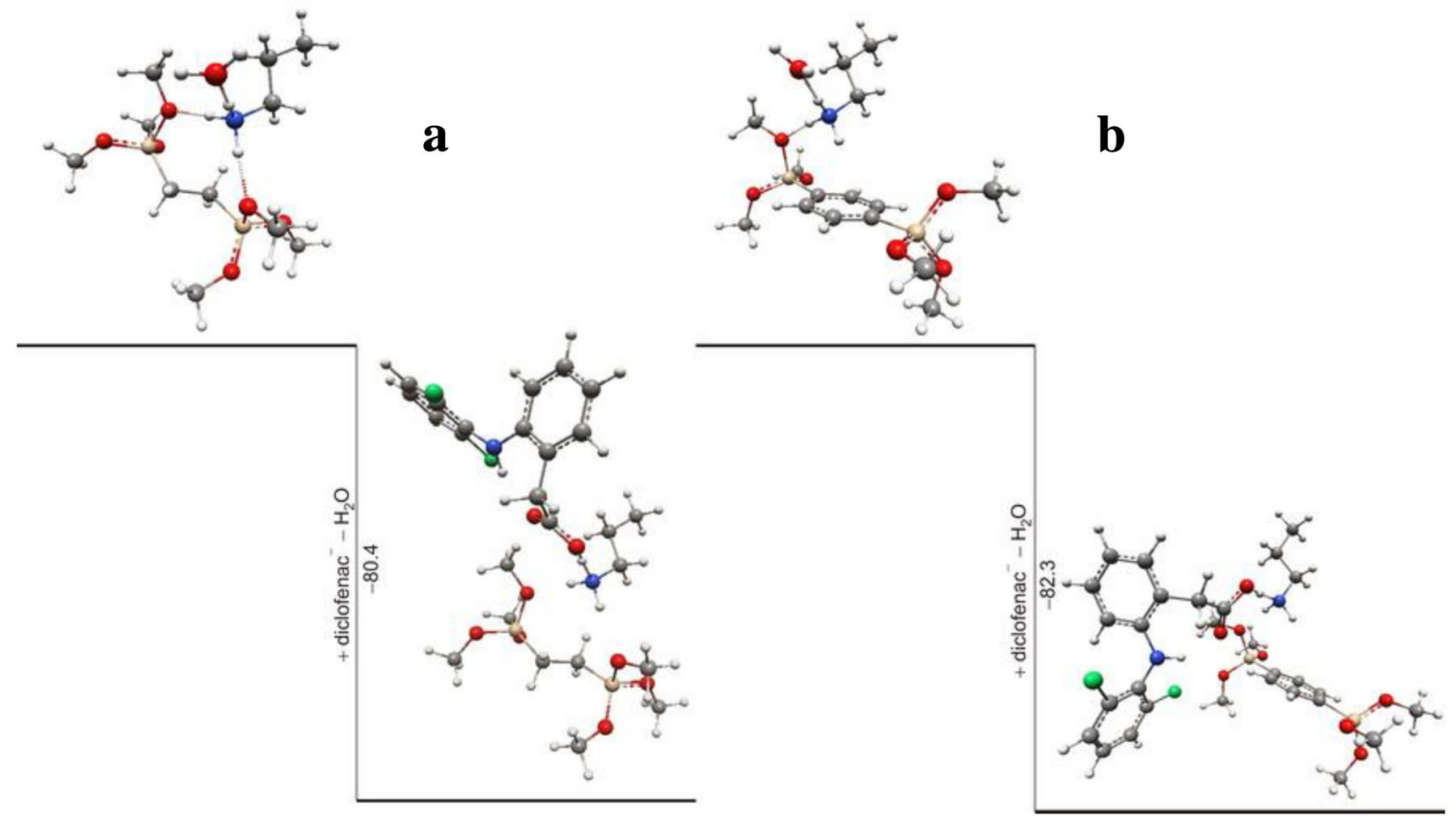

Fig. $10 \mathrm{DICL}^{\ominus}$ interactions with the surface functional groups of the sorbents R2 (a) and R3 (b)

of the reference (Barczak et al. 2018)). In passing we note, that there is yet another possibility of binding $\mathrm{DICL}^{\ominus}$, i.e., via the second oxygen atom of the $\mathrm{COO}^{\ominus}$ group. This does not change the conclusions as far as energetics is concerned.

Another important conclusion coming from the desorption studies is that the addition of bridged monomer markedly affects the releasing rate of DICL, particularly in the time interval 0-300 min. In the case of the samples R3 and $\mathrm{R} 4$ the releasing profile is close to linear in that range without the so-called burst effect (i.e., initial abrupt releasing of the matrix) which is a very desirable feature of any controlled releasing system (CDS). In the literature there is a lot of reports describing applications of silica as CDSs due to their tailored porosity and surface chemistry (Krajnović et al. 2018; Rosenholm and Lindén 2008; Song et al. 2005; Vallet-Regí et al. 2007). Thus the synthesis approach presented here based on co-condensation of three monomers can be applied for more precise tuning of the releasing profiles of the pharmaceuticals from the silica-based CDSs.

Since reusability of any sorbent is one of the crucial parameters bringing it closer to the practical applications, the regenerated samples were used in two next adsorption/desorption cycles to check whether the SSC values will be significantly reduced. The obtained SSC values after three cycles were slightly lower when compared with initial SSC values for fresh samples $(83 \%, 87 \%, 90 \%$ and
$88 \%$, for the samples R1, R2, R3, R4, respectively). Thus all the samples retain over $80 \%$ of their initial adsorption capacity, thus they are suitable for multiple usage. The decrease of SSC values is most probably related to the gradual degradation of porosity and surface chemistry of the samples. This decrease is evident in the case of the sample R1, which testifies that the addition of the bridged co-monomer can positively affect adsorption efficiency of the resulting materials, most probably due to the lower degradation rate of the organically modified silicas (Croissant et al. 2017).

It is interesting to compare the sorption data obtained here with other reports describing adsorption of diclofenac. First it should be noted that, as already shown by us, the uptake amount of other drugs (such as ibuprofen, naproxen or penicillin G) are close to that of DICL due to the similar adsorption mechanism (Barczak 2019). Thus results obtained for DICL can be extended to a wider range of pharmaceuticals. The materials obtained here have higher capacitances than majority of the sorbents reported in the literature (cf. Table 3). It is also worthwhile to mention that such high uptakes observed here are due to two complementary mechanisms: H-bonding and electrostatic interactions. The former is responsible for stronger chemical adsorption and the latter for weaker multilayered physisorption. 
Table 3 Comparison of maximum DICL adsorption capacitances from water of some reported adsorbents

\begin{tabular}{|c|c|c|}
\hline Material & $\begin{array}{l}\text { Observed SSC } \\
\left(\mathrm{mg} \mathrm{g}^{-1}\right)\end{array}$ & References \\
\hline \multicolumn{3}{|l|}{ Carbon sorbents } \\
\hline Graphene & 60 & Jauris et al. (2016) \\
\hline High surface area graphene & 19 & Al-Khateeb et al. (2017) \\
\hline Reduced graphene oxide aerogel & 597 & Hiew et al. (2019) \\
\hline Commercial activated carbon additionally activated with $\mathrm{CO}_{2}$ & 1033 & Moral-Rodríguez et al. (2019) \\
\hline Tea waste derived activated carbon & 62 & Malhotra et al. (2018) \\
\hline Microwave-assisted activated carbon from cocoa shell & 63 & Saucier et al. (2015) \\
\hline Oxidized activated carbon & 487 & Bhadra et al. (2016) \\
\hline \multicolumn{3}{|l|}{ Polymer sorbents } \\
\hline $\begin{array}{l}\text { Magnetic nanoparticles functionalized with poly (styrene-2-acrylamido-2-methyl } \\
\text { propanesulfonic acid) }\end{array}$ & 151 & Hayasi and Saadatjoo (2018) \\
\hline Magnetic amine-functionalized chitosan & 469 & Liang et al. (2019) \\
\hline \multicolumn{3}{|l|}{ Zeolite and MOF-type sorbents } \\
\hline UiO-66- metal-organic-frameworks (MOFs) functionalized with amine groups & 555 & Zhuang et al. (2019) \\
\hline MOF decorated with carboxylate groups & 490 & Luo et al. (2018) \\
\hline Spectrogel type $\mathrm{C}$ commercial organoclay & 42 & Maia et al. (2019) \\
\hline Natural zeolite modified with cetylpyridinium chloride & 22 & Krajišnik et al. (2011) \\
\hline \multicolumn{3}{|l|}{ Silica sorbents } \\
\hline Amine-functionalized templated organobentonites & 601 & Ghemit et al. (2019) \\
\hline Non-functionalized SBA-15 silica & 0.3 & Bui and Choi (2009) \\
\hline Pyridine-functionalized SBA-15 & 631 & Barczak (2018) \\
\hline Bridged mesoporous silicas functionalized with diethylenetriamine groups & $394-842$ & This paper \\
\hline
\end{tabular}

\section{Conclusions}

In this study, a series of functionalized mesoporous organosilicas was synthesized by co-condensation of three different monomers. Addition of bridged monomer (BTESE, BTESB, BTMSD) significantly influences the porosity, surface chemistry and adsorption efficiency of the final materials. The most marked feature of the BTESB- and BTMSD-modified samples is decreased functionalization efficiency indicated by low nitrogen content. The obtained functionalized mesoporous silicas turned out to be promising sorbents for removal of diclofenac. Observed SSC are in the range $394-842 \mathrm{mg} \mathrm{g}^{-1}$ and mostly depend on the number of amine and silanol groups on silica surface. Apart from specific interactions (hydrogen bonding) between the functionalized surface and the drug anions a considerable part of diclofenac is adsorbed by electrostatic forces on the outer surfaces indicating that there is no unique mechanism responsible for its sorption.

Desorption studies showed that releasing profiles are also affected by the type of bridged monomer used. In the case of benzene bridges (BTESB and BTMSD monomers) there is no burst effect in contrast to the sample synthesized without addition of bridged monomer where as much as $30 \%$ of DICL is released in only one minute. This observation makes it possible to use the tailored multi-condensation of different silica precursors as a tool for adjusting controlled desorption properties of the resulting materials.

Acknowledgements This research was supported by the Polish National Science Centre under Grant No. DEC-2012/05/D/ST5/03488 titled Synthesis and characterization of modified organosilica sorbents designed for the sorption of biomolecules. The instrumental characterization of the materials was carried out with the equipment purchased thanks to the financial support of the European Regional Development Fund in the framework of the Polish Innovation Economy Operational Program (contract no. POIG.02.01.00-06-024/09 Center of Functional Nanomaterials).

Open Access This article is distributed under the terms of the Creative Commons Attribution 4.0 International License (http://creativeco mmons.org/licenses/by/4.0/), which permits unrestricted use, distribution, and reproduction in any medium, provided you give appropriate credit to the original author(s) and the source, provide a link to the Creative Commons license, and indicate if changes were made.

\section{References}

Aguado, J., Arsuaga, J.M., Arencibia, A., Lindo, M., Gascón, V.: Aqueous heavy metals removal by adsorption on amine-functionalized mesoporous silica. J. Hazard. Mater. 163, 213-221 (2009). https ://doi.org/10.1016/j.jhazmat.2008.06.080 
Al-Khateeb, L.A., Hakami, W., Salam, M.A.: Removal of non-steroidal anti-inflammatory drugs from water using high surface area nanographene: kinetic and thermodynamic studies. J. Mol. Liq. 241, 733-741 (2017). https://doi.org/10.1016/J.MOLLI Q.2017.06.068

Asefa, T., MacLachlan, M.J., Coombs, N., Ozin, G.A.: Periodic mesoporous organosilicas with organic groups inside the channel walls. Nature 402, 867-871 (1999). https://doi.org/10.1038/47229

Bagheri, E., Ansari, L., Abnous, K., Taghdisi, S.M., Charbgoo, F., Ramezani, M., Alibolandi, M.: Silica based hybrid materials for drug delivery and bioimaging. J. Control. Release 277, 57-76 (2018). https://doi.org/10.1016/J.JCONREL.2018.03.014

Baker, J., Wolinski, K., Malagoli, M., Kinghorn, D., Wolinski, P., Magyarfalvi, G., Saebo, S., Janowski, T., Pulay, P.: Quantum chemistry in parallel with PQS. J. Comput. Chem. 30, 317-335 (2009). https ://doi.org/10.1002/jcc.21052

Barczak, M.: Synthesis and structure of pyridine-functionalized mesoporous SBA-15 organosilicas and their application for sorption of diclofenac. J. Solid State Chem. 258, 232-242 (2018). https://doi.org/10.1016/J.JSSC.2017.10.006

Barczak, M.: Amine-modified mesoporous silicas: morphology-controlled synthesis toward efficient removal of pharmaceuticals. Microporous Mesoporous Mater. 278, 354-365 (2019). https:// doi.org/10.1016/J.MICROMESO.2019.01.012

Barczak, M., Dąbrowski, A., Iwan, M., Rzączyńska, Z.: Mesoporous organosilicas functionalized by alkyl groups: synthesis, structure and adsorption properties. J. Phys. Conf. Ser. 146, 12002 (2009a). https://doi.org/10.1088/1742-6596/146/1/012002

Barczak, M., Pikus, S., Skrzydło-Radomańska, B., Dąbrowski, A.: Synthesis, structure and adsorption properties of nanoporous SBA-15 materials with framework and surface functionalities. Adsorption 15, 278-286 (2009b). https://doi.org/10.1007/s10450-009-9175-8

Barczak, M., Dąbrowski, A., Pikus, S., Ryczkowski, J., Borowski, P., Kozak, M.: Studies of the structure and chemistry of SBA-15 organosilicas functionalized with amine, thiol, vinyl and phenyl groups. Adsorption 16, 457-463 (2010a). https://doi.org/10.1007/ s10450-010-9250-1

Barczak, M., Skwarek, E., Janusz, W., Dabrowski, A., Pikus, S.: Functionalized SBA-15 organosilicas as sorbents of zinc(II) ions. Appl. Surf. Sci. 256, 5370-5375 (2010b). https://doi.org/10.1016/j. apsusc.2009.12.082

Barczak, M., Dobrowolski, R., Dobrzyńska, J., Ziẹba, E., Dạbrowski, A.: Amorphous and ordered organosilicas functionalized with amine groups as sorbents of platinum (II) ions. Adsorption 19, 733-744 (2013). https://doi.org/10.1007/s10450-013-9506-7

Barczak, M., Dobrzyńska, J., Oszust, M., Skwarek, E., Ostrowski, J., Zieba, E., Borowski, P., Dobrowolski, R.: Synthesis and application of thiolated mesoporous silicas for sorption, preconcentration and determination of platinum. Mater. Chem. Phys. 181, 126-135 (2016). https://doi.org/10.1016/j.matchemphys.2016.06.042

Barczak, M., Wierzbicka, M., Borowski, P.: Sorption of diclofenac onto functionalized mesoporous silicas: experimental and theoretical investigations. Microporous Mesoporous Mater. 264, 254-264 (2018). https://doi.org/10.1016/J.MICROMESO.2018.01.013

Beck, J.S., Vartuli, J.C., Roth, W.J., Leonowicz, M.E., Kresge, C.T., Schmitt, K.D., Chu, C.T.W., Olson, D.H., Sheppard, E.W., McCullen, S.B., Higgins, J.B., Schlenker, J.L.: A new family of mesoporous molecular sieves prepared with liquid crystal templates. J. Am. Chem. Soc. 114, 10834-10843 (1992). https://doi. org/10.1021/ja00053a020

Becke, A.D.: Density-functional thermochemistry. III. The role of exact exchange. J. Chem. Phys. 98, 5648-5652 (1993). https:// doi.org/10.1063/1.464913
Bhadra, B.N., Seo, P.W., Jhung, S.H.: Adsorption of diclofenac sodium from water using oxidized activated carbon. Chem. Eng. J. 301, 27-34 (2016). https://doi.org/10.1016/j.cej.2016.04.143

Bruzzoniti, M.C., Prelle, A., Sarzanini, C., Onida, B., Fiorilli, S., Garrone, E.: Retention of heavy metal ions on SBA-15 mesoporous silica functionalised with carboxylic groups. J. Sep. Sci. 30, 2414 2420 (2007). https://doi.org/10.1002/jssc.200700182

Bui, T.X., Choi, H.: Adsorptive removal of selected pharmaceuticals by mesoporous silica SBA-15. J. Hazard. Mater. 168, 602-608 (2009). https://doi.org/10.1016/j.jhazmat.2009.02.072

Cashin, V.B., Eldridge, D.S., Yu, A., Zhao, D.: Surface functionalization and manipulation of mesoporous silica adsorbents for improved removal of pollutants: a review. Environ. Sci. Water Res. Technol. 4, 110-128 (2018). https://doi.org/10.1039/C7EW0 0322F

Chang, F.-Y., Chao, K.-J., Cheng, H.-H., Tan, C.-S.: Adsorption of $\mathrm{CO}_{2}$ onto amine-grafted mesoporous silicas. Sep. Purif. Technol. 70, 87-95 (2009). https://doi.org/10.1016/j.seppur.2009.08.016

Chong, S.M., Zhao, X.S.: Functionalization of SBA-15 with APTES and characterization of functionalized materials. J. Phys. Chem. B 107, 12650-12657 (2003). https://doi.org/10.1021/jp035 $877 \& \% 23 \times 002 \mathrm{~B}$

Cotea, V.V., Luchian, C.E., Bilba, N., Niculaua, M.: Mesoporous silica SBA-15, a new adsorbent for bioactive polyphenols from red wine. Anal. Chim. Acta 732, 180-185 (2012). https://doi. org/10.1016/J.ACA.2011.10.019

Croissant, J.G., Fatieiev, Y., Khashab, N.M.: Degradability and clearance of silicon, organosilica, silsesquioxane, silica mixed oxide, and mesoporous silica nanoparticles. Adv. Mater. 29, 1604634 (2017). https://doi.org/10.1002/adma.201604634

Croissant, J.G., Fatieiev, Y., Almalik, A., Khashab, N.M.: Mesoporous silica and organosilica nanoparticles: physical chemistry, biosafety, delivery strategies, and biomedical applications. Adv. Healthc. Mater. 7, 1700831 (2018). https://doi.org/10.1002/ adhm. 201700831

Crudden, C.M., Sateesh, M., Lewis, R.: Mercaptopropyl-modified mesoporous silica: a remarkable support for the preparation of a reusable, heterogeneous palladium catalyst for coupling reactions. J. Am. Chem. Soc. 127, 10045-10050 (2005). https://doi. org/10.1021/JA0430954

Da'na, E.: Adsorption of heavy metals on functionalized-mesoporous silica: a review. Microporous Mesoporous Mater. 247, 145-157 (2017). https://doi.org/10.1016/J.MICROMESO.2017.03.050

Dobrowolski, R., Oszust-Cieniuch, M., Dobrzyńska, J., Barczak, M.: Amino-functionalized SBA-15 mesoporous silicas as sorbents of platinum (IV) ions. Colloids Surf. A 435, 63-70 (2013). https:// doi.org/10.1016/j.colsurfa.2012.12.001

Fan, J., Wang, X., Teng, W., Yang, J., Ran, X., Gou, X., Bai, N., Lv, M., Xu, H., Li, G., Zhang, W., Zhao, D.: Phenyl-functionalized mesoporous silica materials for the rapid and efficient removal of phthalate esters. J. Colloid Interface Sci. 487, 354-359 (2017). https://doi.org/10.1016/j.jcis.2016.10.042

Frisch, M.J., Pople, J.A., Binkley, J.S.: Self-consistent molecular orbital methods 25. Supplementary functions for Gaussian basis sets. J. Chem. Phys. 80, 3265-3269 (1984). https://doi. org/10.1063/1.447079

Garcia-Bennet, A.E., Hodgkins, R.P., Sen, T., Anderson, M.W., Wright, P.A.: HRTEM imaging of mesoporous phase transition from hexagonal P6mm to cubic La 3d symmetry. Stud. Surf. Sci. Catal. 154, 400-407 (2004)

Ghemit, R., Makhloufi, A., Djebri, N., Flilissa, A., Zerroual, L., Boutahala, M.: Adsorptive removal of diclofenac and ibuprofen from aqueous solution by organobentonites: study in single and binary systems. Groundw. Sustain. Dev. 8, 520-529 (2019). https://doi. org/10.1016/J.GSD.2019.02.004 
Gunathilake, C., Manchanda, A.S., Ghimire, P., Kruk, M., Jaroniec, M.: Amine-modified silica nanotubes and nanospheres: synthesis and $\mathrm{CO}_{2}$ sorption properties. Environ. Sci. Nano. 3, 806-817 (2016). https://doi.org/10.1039/C6EN00125D

Han, L., Sakamoto, Y., Terasaki, O., Li, Y., Che, S.: Synthesis of carboxylic group functionalized mesoporous silicas (CFMSs) with various structures. J. Mater. Chem. 17, 1216 (2007). https://doi. org/10.1039/b615209k

Hayasi, M., Saadatjoo, N.: Preparation of magnetic nanoparticles functionalized with poly (styrene-2-acrylamido-2-methyl propanesulfonic acid) as novel adsorbents for removal of pharmaceuticals from aqueous solutions. Adv. Polym. Technol. 37, 1941-1953 (2018). https://doi.org/10.1002/adv.21852

Hiew, B.Y.Z., Lee, L.Y., Lai, K.C., Gan, S., Thangalazhy-Gopakumar, S., Pan, G.-T., Yang, T.C.-K.: Adsorptive decontamination of diclofenac by three-dimensional graphene-based adsorbent: response surface methodology, adsorption equilibrium, kinetic and thermodynamic studies. Environ. Res. 168, 241-253 (2019). https://doi.org/10.1016/J.ENVRES.2018.09.030

Huang, C.-H., Chang, K.-P., Ou, H.-D., Chiang, Y.-C., Wang, C.-F.: Adsorption of cationic dyes onto mesoporous silica. Microporous Mesoporous Mater. 141, 102-109 (2011). https://doi. org/10.1016/J.MICROMESO.2010.11.002

Huang, D., Sha, Y., Zheng, S., Liu, B., Deng, C.: Preparation of phenyl group-functionalized magnetic mesoporous silica microspheres for fast extraction and analysis of acetaldehyde in mainstream cigarette smoke by gas chromatography-mass spectrometry. Talanta 115, 427-434 (2013). https://doi.org/10.1016/J.TALAN TA.2013.05.068

Inagaki, S., Guan, S., Ohsuna, T., Terasaki, O.: An ordered mesoporous organosilica hybrid material with a crystal-like wall structure. Nature 416, 304-307 (2002). https://doi.org/10.1038/416304a

Jaiboon, V., Yoosuk, B., Prasassarakich, P.: Amine modified silica xerogel for $\mathrm{H}_{2} \mathrm{~S}$ removal at low temperature. Fuel Process. Technol. 128, 276-282 (2014). https://doi.org/10.1016/J.FUPRO C.2014.07.032

Jauris, I.M., Matos, C.F., Saucier, C., Lima, E.C., Zarbin, A.J.G., Fagan, S.B., Machado, F.M., Zanella, I., Park, C.R., Tascon, J.M.: Adsorption of sodium diclofenac on graphene: a combined experimental and theoretical study. Phys. Chem. Chem. Phys. 18, 1526-1536 (2016). https://doi.org/10.1039/C5CP05940B

Katiyar, A., Smirniotis, P., Pinto, N.G.: Functionalized nanoporous molecular sieves for chromatographic separations of proteins. Presented at the 2005 Annual Meeting: 3 November 2005

Kecht, J., Schlossbauer, A., Bein, T.: Selective functionalization of the outer and inner surfaces in mesoporous silica nanoparticles. Chem. Mater. 20, 7207-7214 (2008). https://doi.org/10.1021/ cm801484r

Krajišnik, D., Daković, A., Milojević, M., Malenović, A., Kragović, M., Bogdanović, D.B., Dondur, V., Milić, J.: Properties of diclofenac sodium sorption onto natural zeolite modified with cetylpyridinium chloride. Colloids Surf. B 83, 165-172 (2011). https://doi.org/10.1016/J.COLSURFB.2010.11.024

Krajnović, T., Maksimović-Ivanić, D., Mijatović, S., Drača, D., Wolf, K., Edeler, D., Wessjohann, L.A., Kaluđerović, G.N.: Drug delivery system for emodin based on mesoporous silica SBA-15. Nanomaterials (Basel, Switzerland) 8, 322 (2018). https://doi. org/10.3390/nano8050322

Kresge, C.T., Leonowicz, M.E., Roth, W.J., Vartuli, J.C., Beck, J.S.: Ordered mesoporous molecular sieves synthesized by a liquidcrystal template mechanism. Nature 359, 710-712 (1992). https ://doi.org/10.1038/359710a0

Krishnan, R., Binkley, J.S., Seeger, R., Pople, J.A.: Self-consistent molecular orbital methods. XX. A basis set for correlated wave functions. J. Chem. Phys. 72, 650-654 (1980). https://doi. org/10.1063/1.438955
Li, X., Shi, B., Li, M., Mao, L.: Synthesis of highly ordered alkylfunctionalized mesoporous silica by co-condensation method and applications in surface coating with superhydrophilic/antifogging properties. J. Porous Mater. 22, 201-210 (2015). https ://doi.org/10.1007/s10934-014-9886-4

Liang, X.X., Omer, A.M., Hu, Z., Wang, Y., Yu, D., Ouyang, X.: Efficient adsorption of diclofenac sodium from aqueous solutions using magnetic amine-functionalized chitosan. Chemosphere 217, 270-278 (2019). https://doi.org/10.1016/J.CHEMO SPHERE.2018.11.023

Liu, A.M., Hidajat, K., Kawi, S., Zhao, D.Y., Czernuszewicz, R.S., Kevan, L., Stucky, G.D.: A new class of hybrid mesoporous materials with functionalized organic monolayers for selective adsorption of heavy metal ions. Chem. Commun. 11, 11451146 (2000). https://doi.org/10.1039/b0026611

Liu, X., Tian, B., Yu, C., Gao, F., Xie, S., Tu, B., Che, R., Peng, L.-M., Zhao, D.: Room-temperature synthesis in acidic media of large-pore three-dimensional bicontinuous mesoporous silica with Ia3d symmetry. Angew. Chem. Int. Ed. 41, 38763878 (2002). https://doi.org/10.1002/1521-3773(20021 018)41:20\%3C3876::AID-ANIE3876\%3E3.0.CO;2-R

Luo, Z., Fan, S., Liu, J., Liu, W., Shen, X., Wu, C., Huang, Y., Huang, G., Huang, H., Zheng, M., Luo, Z., Fan, S., Liu, J., Liu, W., Shen, X., Wu, C., Huang, Y., Huang, G., Huang, H., Zheng, M.: A 3D stable metal-organic framework for highly efficient adsorption and removal of drug contaminants from water. Polymers (Basel) 10, 209 (2018). https://doi.org/10.3390/ polym 10020209

Maia, G.S., de Andrade, J.R., da Silva, M.G.C., Vieira, M.G.A.: Adsorption of diclofenac sodium onto commercial organoclay: kinetic, equilibrium and thermodynamic study. Powder Technol. 345, 140-150 (2019). https://doi.org/10.1016/J.POWTE C.2018.12.097

Malhotra, M., Suresh, S., Garg, A.: Tea waste derived activated carbon for the adsorption of sodium diclofenac from wastewater: adsorbent characteristics, adsorption isotherms, kinetics, and thermodynamics. Environ. Sci. Pollut. Res. 25, 32210-32220 (2018). https ://doi.org/10.1007/s11356-018-3148-y

Martín, A., Morales, V., Ortiz-Bustos, J., Pérez-Garnes, M., Bautista, L.F., García-Muñoz, R.A., Sanz, R.: Modelling the adsorption and controlled release of drugs from the pure and amino surfacefunctionalized mesoporous silica hosts. Microporous Mesoporous Mater. 262, 23-34 (2018). https://doi.org/10.1016/J.MICRO MESO.2017.11.009

Martucci, A., Pasti, L., Marchetti, N., Cavazzini, A., Dondi, F., Alberti, A.: Adsorption of pharmaceuticals from aqueous solutions on synthetic zeolites. Microporous Mesoporous Mater. 148, 174-183 (2012). https://doi.org/10.1016/j.micromeso.2011.07.009

McManamon, C., Burke, A.M., Holmes, J.D., Morris, M.A.: Aminefunctionalised SBA-15 of tailored pore size for heavy metal adsorption. J. Colloid Interface Sci. 369, 330-337 (2012). https ://doi.org/10.1016/j.jcis.2011.11.063

Mizoshita, N., Tani, T., Inagaki, S.: Syntheses, properties and applications of periodic mesoporous organosilicas prepared from bridged organosilane precursors. Chem. Soc. Rev. 40, 789-800 (2011). https://doi.org/10.1039/C0CS00010H

Moral-Rodríguez, A.I., Leyva-Ramos, R., Ania, C.O., Ocampo-Pérez, R., Isaacs-Páez, E.D., Carrales-Alvarado, D.H., Parra, J.B.: Tailoring the textural properties of an activated carbon for enhancing its adsorption capacity towards diclofenac from aqueous solution. Environ. Sci. Pollut. Res. (2019). https://doi.org/10.1007/s1135 6-018-3991-x

Mureseanu, M., Reiss, A., Stefanescu, I., David, E., Parvulescu, V., Renard, G., Hulea, V.: Modified SBA-15 mesoporous silica for heavy metal ions remediation. Chemosphere 73, 1499-1504 (2008). https://doi.org/10.1016/j.chemosphere.2008.07.039 
Rosenholm, J.M., Lindén, M.: Towards establishing structure-activity relationships for mesoporous silica in drug delivery applications. J. Control. Release 128, 157-164 (2008). https://doi. org/10.1016/j.jconrel.2008.02.013

Rosenholm, J.M., Czuryszkiewicz, T., Kleitz, F., Rosenholm, J.B., Lindén, M.: On the nature of the Brønsted acidic groups on native and functionalized mesoporous siliceous SBA-15 as studied by benzylamine adsorption from solution. Langmuir 23, 4315-4323 (2007). https://doi.org/10.1021/la062450w

Saito, A., Foley, H.C.: Curvature and parametric sensitivity in models for adsorption in micropores. AIChE J. 37, 429-436 (1991). https ://doi.org/10.1002/aic.690370312

Sanaeishoar, H., Sabbaghan, M., Mohave, F.: Synthesis and characterization of micro-mesoporous MCM-41 using various ionic liquids as co-templates. Microporous Mesoporous Mater. 217, 219-224 (2015). https://doi.org/10.1016/J.MICROMESO.2015.06.027

Saucier, C., Adebayo, M.A., Lima, E.C., Cataluña, R., Thue, P.S., Prola, L.D.T., Puchana-Rosero, M.J., Machado, F.M., Pavan, F.A., Dotto, G.L.: Microwave-assisted activated carbon from cocoa shell as adsorbent for removal of sodium diclofenac and nimesulide from aqueous effluents. J. Hazard. Mater. 289, 18-27 (2015). https://doi.org/10.1016/j.jhazmat.2015.02.026

Schmidt-Winkel, P., Lukens, W.W., Zhao, D., Yang, P., Chmelka, B.F., Stucky, G.D.: Mesocellular siliceous foams with uniformly sized cells and windows. J. Am. Chem. Soc. 121, 254-255 (1999). https ://doi.org/10.1021/JA983218I

Shen, S., Chow, P.S., Kim, S., Zhu, K., Tan, R.B.H.: Synthesis of carboxyl-modified rod-like SBA-15 by rapid co-condensation. J. Colloid Interface Sci. 321, 365-372 (2008). https://doi.org/10.1016/j. jcis.2008.02.020

Song, S.W., Hidajat, K., Kawi, S.: Functionalized SBA-15 materials as carriers for controlled drug delivery: influence of surface properties on matrix-drug interactions. Langmuir 21, 9568-9575 (2005). https://doi.org/10.1021/la051167e

Toufaily, J., Koubaissy, B., Kafrouny, L., Hamad, H., Magnoux, P., Ghannam, L., Karout, A., Hazimeh, H., Nemra, G., Hamieh, M., Ajouz, N., Hamieh, T.: Functionalization of SBA-15 materials for the adsorption of phenols from aqueous solution. Open Eng. 3, 126-134 (2013). https://doi.org/10.2478/s13531-012-0038-9

Trindade, F.J., Rey, J.F.Q., Brochsztain, S.: Modification of molecular sieves MCM-41 and SBA-15 with covalently grafted pyromellitimide and 1,4,5,8-naphthalenediimide. J. Colloid Interface Sci. 368, 34-40 (2012). https://doi.org/10.1016/J.JCIS.2011.10.071

Tsai, C.H., Chang, W.C., Saikia, D., Wu, C.E., Kao, H.M.: Functionalization of cubic mesoporous silica SBA-16 with carboxylic acid via one-pot synthesis route for effective removal of cationic dyes. J. Hazard. Mater. 309, 236-248 (2016). https://doi.org/10.1016/j. jhazmat.2015.08.051

Vallet-Regí, M., Balas, F., Arcos, D.: Mesoporous materials for drug delivery. Angew. Chem. Int. Ed. 46, 7548-7558 (2007). https:// doi.org/10.1002/anie.200604488

Vallet-Regí, M., Colilla, M., Izquierdo-Barba, I., Manzano, M.: Mesoporous silica nanoparticles for drug delivery: current insights. Molecules 23, 47 (2017). https://doi.org/10.3390/molec ules 23010047
Visuvamithiran, P., Palanichamy, M., Shanthi, K., Murugesan, V.: Selective epoxidation of olefins over Co(II)-Schiff base immobilised on KIT-6. Appl. Catal. A 462-463, 31-38 (2013). https:// doi.org/10.1016/J.APCATA.2013.05.007

Walcarius, A.: Silica-based electrochemical sensors and biosensors: recent trends. Curr. Opin. Electrochem. 10, 88-97 (2018). https ://doi.org/10.1016/J.COELEC.2018.03.017

Wang, Y., Zibrowius, B., Yang, C., Spliethoff, B., Schüth, F.: Synthesis and characterization of large-pore vinyl-functionalized mesoporous silica SBA-15. Chem. Commun. 0, 46-47 (2004). https://doi.org/10.1039/B309578A

Yasmin, T., Müller, K.: Synthesis and characterization of surface modified SBA-15 silica materials and their application in chromatography. J. Chromatogr. A 1218, 6464-6475 (2011). https://doi. org/10.1016/j.chroma.2011.07.035

Yokoi, T., Yoshitake, H., Tatsumi, T., Onida, B., Rocchia, M., Nagy, J.B., Macquarrie, D.J., Blanc, A.C., Fajula, F., McCullen, S.B., Higgins, J.B., Schlenker, J.L.: Synthesis of amino-functionalized MCM-41 via direct co-condensation and post-synthesis grafting methods using mono-, di- and tri-amino-organoalkoxysilanes. Chem. Mater. 15, 1132-1139 (2003). https://doi.org/10.1039/ B310576H

Yoshina-Ishii, C., Asefa, T., Coombs, N., MacLachlan, M.J., Ozin, G.A.: Periodic mesoporous organosilicas, PMOs: fusion of organic and inorganic chemistry 'inside' the channel walls of hexagonal mesoporous silica. Chem. Commun. 0, 2539-2540 (1999). https://doi.org/10.1039/a908252b

Zhang, L., Liu, J., Yang, J., Yang, Q., Li, C.: Direct synthesis of highly ordered amine-functionalized mesoporous ethane-silicas. Microporous Mesoporous Mater. 109, 172-183 (2008). https:// doi.org/10.1016/j.micromeso.2007.04.050

Zhao, D., Feng, J., Huo, Q., Melosh, N., Fredrickson, G.H., Chmelka, B.F., Stucky, G.D.: Triblock copolymer syntheses of mesoporous silica with periodic 50 to 300 angstrom pores. Science 279, 548552 (1998)

Zhao, J., Gao, F., Fu, Y., Jin, W., Yang, P., Zhao, D., Stucky, G.D.: Biomolecule separation using large pore mesoporous SBA-15 as a substrate in high performance liquid chromatography. Chem. Commun. 73, 752-753 (2002). https://doi.org/10.1039/b110637f

Zhu, Y., Li, H., Zheng, Q., Xu, J., Li, X.: Amine-functionalized SBA15 with uniform morphology and well-defined mesostructure for highly sensitive chemosensors to detect formaldehyde vapor. Langmuir 28, 7843-7850 (2012). https://doi.org/10.1021/la300 $560 \mathrm{j}$

Zhuang, S., Cheng, R., Wang, J.: Adsorption of diclofenac from aqueous solution using UiO-66-type metal-organic frameworks. Chem. Eng. J. 359, 354-362 (2019). https://doi.org/10.1016/J. CEJ.2018.11.150

Publisher's Note Springer Nature remains neutral with regard to jurisdictional claims in published maps and institutional affiliations. 